\title{
Path Dependence of the Quark Nonlocal Condensate within the Instanton Model
}

\author{
L. A. Trevisan ${ }^{1,3}$, A. E. Dorokhov ${ }^{2}$, and Lauro Tomio ${ }^{3}$ \\ ${ }^{1}$ Departamento de Matemática, Universidade Estadual de Ponta Grossa, \\ Av. Carlos Cavalcante, 4748, 84030-000, Ponta Grossa, PR, Brazil \\ ${ }^{2}$ Bogoliubov Laboratory of Theoretical Physics, JINR, Dubna 141980, Russia \\ ${ }^{3}$ Instituto de Física Teórica, Universidade Estadual Paulista, Rua Pamplona 145, 01405-900, São Paulo, SP, Brazil
}

Received on 4 October, 2003

\begin{abstract}
Within the instanton liquid model, we study the dependence of the gauge invariant two-point quark correlator on the path used to perform the color parallel transport between two points in the Euclidean space.
\end{abstract}

\section{Introduction}

The nonlocal vacuum condensates or vacuum correlators describe the distribution of quarks and gluons in the nonperturbative QCD vacuum [1]. Physically, it means that vacuum quarks and gluons can flow through the vacuum with nonzero momentum. Nonlocal condensates play an important role in hadron physics. The nonlocal properties of the vacuum condensates are of principle importance in the study of the distribution functions of quarks and gluons in QCD vacuum and in hadrons $[1,2]$.

In general, the gauge-invariant (and path independent) nonlocal quark-quark correlator is defined by

$$
\mathcal{M}(x, y)=\sum_{\mathcal{C}} e^{-L[\mathcal{C}]} \mathcal{M}^{\mathcal{C}}(x, y)
$$

where

$$
\begin{aligned}
& \mathcal{M}^{\mathcal{C}}(x, y)=\left(1 / N_{c}\right) \times \\
& \left\langle 0\left|: \operatorname{Tr}\left\{\mathrm{P} \exp \left(i g \int_{y, \mathcal{C}}^{x} d z^{\mu} A_{\mu}^{a}(z) T^{a}\right) q(x) \bar{q}(y)\right\}:\right| 0\right\rangle
\end{aligned}
$$

is the quark correlator with the Schwinger phase factor pathordered along any path $\mathcal{C}$ from 0 to $x$. The P-exponential ensures the parallel transport of color from one point to another. $T^{a}$ are the matrices of the algebra of the color group $\mathrm{SU}\left(\mathrm{N}_{c}\right)$ in the fundamental representation. The trace is taken on the color indices and $\exp \{-L[\mathcal{C}]\}$ is the dynamical weight of each path configuration.

To evaluate Eq. (1), one has to calculate the nonperturbative expectation value of the Wilson line operator for an arbitrary path $\mathcal{C}$ and perform resummation over all possible path configurations, pictorically represented in Fig. 1. Both tasks are extremely difficult and cannot be performed in full manner at the current stage. In the field theory there is a special class of observables for which the straight-line paths are dominated and the path integrations can be performed exactly. We can mention two examples: propagation of an energetic quark through a cloud of soft gluons, and the behaviour of light quark in the field of infinitely heavy quark. Such an approximation is called the straight-line path approximation [3].

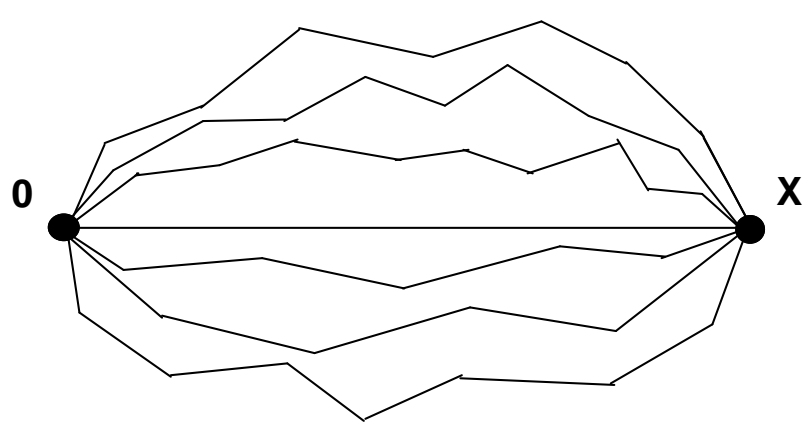

Figure 1. Schematic representation of the quark correlator with arbitrary paths between the quarks, which are in the positions 0 and $x$.

Next, we describe the so called straight-line path approximation. In section III, we discuss the path dependence and present the formalism we are considering. In section IV we present our results and conclusions.

\section{Straight-line path approximation}

The gauge-invariant correlators $\mathcal{M}^{\mathcal{C}}(x, y)$ in (1) in principle depend on the choice of the path $\mathcal{C}$. Usually, the path $\mathcal{C}$ appearing in Eq. (2) is assumed to be a straight line connecting the points 0 and $x$. With the path, that is a straight line connecting the points 0 and $x$, the Schwinger string (2) reads:

$$
S(0, x)=\mathrm{P} \exp \left(i g \int_{0}^{1} d t x^{\mu} A_{\mu}(x t)\right) .
$$


Then, in the Euclidean region, the translational, $\mathrm{O}(4)$ and parity symmetries require the correlator (2) to be of the following form:

$$
\mathcal{M}^{(\mathcal{C})}(x, 0)=\langle 0|: \bar{q}(0) q(0):| 0\rangle \mathcal{Q}^{(\mathcal{C})}\left(x^{2}\right)
$$

where $\mathcal{Q}$ is an invariant function of $x^{2}$. In principle, every choice of a path $\mathcal{C}_{[0, x]}$ connecting the two points 0 and $x$ in the expression (2) for the Schwinger string operator will generate a different quark correlator, that we have denoted in Eq. $(1)$ as $\mathcal{Q}^{(\mathcal{C})}(x)\left[\mathcal{Q}^{(\mathcal{C})}(0)=1\right]$.

Recently, the quark nonlocal condensate, with the straight-line parallel transport, has been evaluated on the lattice [4] and semi-classically in the instanton dilute-liquid model [5]. The instanton model of the QCD vacuum is a realistic one in prediction of the behaviour of the quark and gluon correlation functions [5, 2]. For the straight line path the averaging over the instanton vacuum gives in the singleinstanton approximation the result $[5,2]$

$$
\begin{aligned}
\mathcal{Q}_{I}\left(x^{2}\right) & =\frac{8 \rho^{2}}{\pi} \int_{0}^{\infty} d r r^{2} \int_{-\infty}^{\infty} d t \times \\
& \times \frac{\cos \left[\frac{r}{R}\left[\arctan \left(\frac{t+|x|}{R}\right)-\arctan \left(\frac{t}{R}\right)\right]\right]}{\left[R^{2}+t^{2}\right]^{3 / 2}\left[R^{2}+(t+|x|)^{2}\right]^{3 / 2}},
\end{aligned}
$$

where $\rho$ is the average instanton size and $R=\sqrt{\rho^{2}+r^{2}}$.

In the next, we discuss the path dependence of the correlators and present the formalism that we are considering.

\section{Path dependence of the nonlocal quark condensate}

The nonlocal quark condensate with straight-line path has a direct physical interpretation in the heavy quark theory of heavy-light mesons as it describes the propagation of a light quark in the color field of an infinitely heavy quark. In the light quark sector one needs to know the path dependence of the correlator and average over all possibilities, as shown in Fig. 1. Moreover, knowledge of the path dependence is also important in modeling of the higher dimensional nonlocal condensates used in QCD sum rules for hadron wave functions.

No analysis exists of what happens for a different choice of the path $\mathcal{C}$ other than the straight line, except recent very intersting results on the path dependence of the gaugeinvariant gluon field strength correlator studied in numerical simulations on a lattice in Ref. [7]. Here, we report our results on the path dependence of the gauge-invariant quark two-point correlator within the instanton vacuum model for arbitrary paths made of two joined straight lines with cusp (polygon path). The broken-line quark condensate has the general structure (see, e.i. $[8,9]$ )

$$
\begin{aligned}
\mathcal{M}^{\mathcal{C}}(x, y) & =\langle\bar{q} q\rangle\left[\mathcal{Q}_{S}^{(\mathcal{C})}(x, y)+\frac{\lambda_{q}^{2}}{24} \mathcal{Q}_{T}^{(\mathcal{C})}(x, y)[\hat{x}, \hat{y}]\right. \\
& \left.-\frac{i}{4}\left(\mathcal{Q}_{V}^{(\mathcal{C})}(x, y) \hat{x}-\mathcal{Q}_{V}^{(\mathcal{C})}(y, x) \hat{y}\right)\right]
\end{aligned}
$$

where $\lambda_{q}^{2}$ is an average quark virtuality in the QCD vacuum.

In order to calculate the matrix element (2) we use the instanton liquid model. In the single instanton approximation the vacuum expectation value is found by taking the classical instanton solution and quark zero modes developed in its field and averaging over instanton collective variables: its size, its color orientation and its position in space. Then one gets

$$
\begin{aligned}
& Q_{I, i}^{(\mathcal{C})}(x)=\int d D(\rho) \int d U \int d z_{0}^{4} \frac{1}{N_{c}} \operatorname{Tr}\left\{\bar{q}_{0}\left(x-z_{0}\right) \Gamma_{i}\right. \\
& \left.\times P \exp \left[-i g \int_{x}^{y} d z_{\mu} A_{I \mu}^{a}\left(z-z_{0}\right) \frac{\tau^{a}}{2}\right] q_{0}\left(y-z_{0}\right)\right\},
\end{aligned}
$$

where the instanton field in the regular gauge is given by

$$
A_{I \mu}^{a}(z)=\frac{1}{g} \eta_{\mu \nu}^{a} z_{\nu} \Phi(z), \quad \Phi(z)=\frac{2}{\rho^{2}+z^{2}},
$$

and corresponding quark zero mode

$$
q_{0}(z)=\frac{\rho}{\pi} \frac{1}{\left(\rho^{2}+z^{2}\right)^{3 / 2}} \chi, \quad \bar{\chi} \chi=2 .
$$

In Eq. (8), $\eta_{\mu \nu}^{a}$ is the antisymmetric 't Hooft symbol. The broken-line path is parameterized in the form

$$
\mathcal{C}_{[y, 0, x]}=\left\{\begin{array}{l}
y_{\mu} \lambda, \quad-1 \leq \lambda \leq 0, \\
x_{\mu} \lambda^{\prime}, \quad 0 \leq \lambda^{\prime} \leq 1,
\end{array}\right.
$$

with the cusp angle $\alpha$ defined as

$$
\cos (\alpha)=\frac{y \cdot x}{|x||y|}
$$

Note, that in the zero mode approximation, $\mathcal{Q}_{V}^{(\mathcal{C})}(x, y)$ is absent due to its odd chirality structure. Let us consider the scalar structure, $\mathcal{Q}_{S}^{(\mathcal{C})}(x, y)$, in Eq. (6). The final result, which is gauge and translation invariant, is given by

$$
\begin{aligned}
& Q_{S, \mathrm{I}}^{(\mathcal{C})}(y, x)=n_{c} \int d z_{0}^{4} \bar{q}_{0}\left(y-z_{0}\right) q_{0}\left(x-z_{0}\right) \times \\
& \times\left\{\cos \left[\alpha_{y}\right] \cos \left[\alpha_{x}\right]+\left(n_{y}, n_{x}\right) \sin \left[\alpha_{y}\right] \sin \left[\alpha_{x}\right]\right\},
\end{aligned}
$$

where the corresponding phase factors $\alpha_{x, y}$ and unit (in color space) vectors $n_{x, y}^{a}\left(n_{x, y}^{2}=1\right)$ are given by

$$
\begin{aligned}
\alpha_{u} & =\frac{1}{2} \sqrt{u^{2} z_{0}^{2}-\left(u z_{0}\right)^{2}} \int_{0}^{1} d \lambda \Phi\left(u \lambda-z_{0}\right), \\
n_{u}^{a} & =\frac{u_{\mu} \eta_{\mu \nu}^{a} z_{0 \nu}}{\sqrt{u^{2} z_{0}^{2}-\left(u z_{0}\right)^{2}}}
\end{aligned}
$$


with $u=x$ or $y$. The scalar product in color space is

$$
\left(n_{y}, n_{x}\right)=\frac{(y, x) z_{0}^{2}-\left(z_{0}, y\right)\left(x, z_{0}\right)}{\sqrt{y^{2} z_{0}^{2}-\left(y z_{0}\right)^{2}} \sqrt{x^{2} z_{0}^{2}-\left(x z_{0}\right)^{2}}} .
$$

In the next section, we present and discuss the results of our calculations.

\section{Results and conclusions}

We have explored the dependence on the path by calculating the correlator, considering various different paths, as shown in Figs. 2 and 4. The corresponding results are presented in Figs. 3 and 5. All distances are given in units of the instanton size $\rho$, i.e., for $\rho=1$.

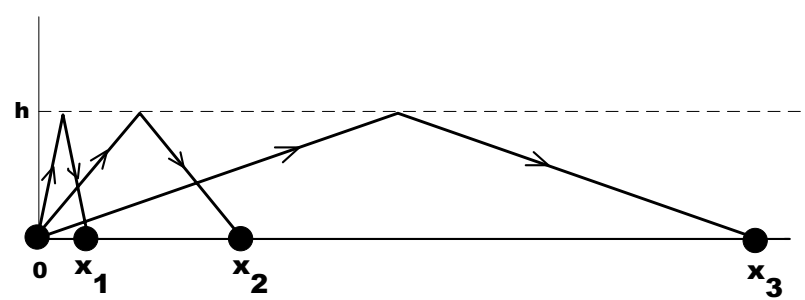

Figure 2. Different paths between quarks that are at fixed deflection from the straight line. The position of the cusp is at equal distance from each quark.

In Fig. 2, we show a set of paths with fixed transverse size $h$ parameterizing the deviation from the straight-line path. A similar set of paths has been considered in lattice calculations [7] for the gluon field-strength correlator. We have chosen the position of the cusp in the broken-line path in Fig. 2 in the middle of the line $(0, x)$. All quark correlators are normalized at zero distance, $x=0$, independently on the form of closed path. All curves in Fig. 3 have the same large $x$ asymptotics. This is because at large distances the relative deflection $h$ becomes very small $(h<<x)$ and the path becomes almost indistinguished from the straightline path.

The same considerations also apply to the sets of paths shown in Fig. 4, for a fixed distance between quarks and equal distance of each cusp from the quarks. This set of paths, with fixed equal distances from the cusps to the quarks, shows the maximal effects related to the deflection of the broken-line path from the straight-line one. The results for the correlator $Q(x, h)$ are shown in Fig. 3, versus the distance $x$; and, in Fig. 5, versus the distances $h$, related to the angle $\alpha$.

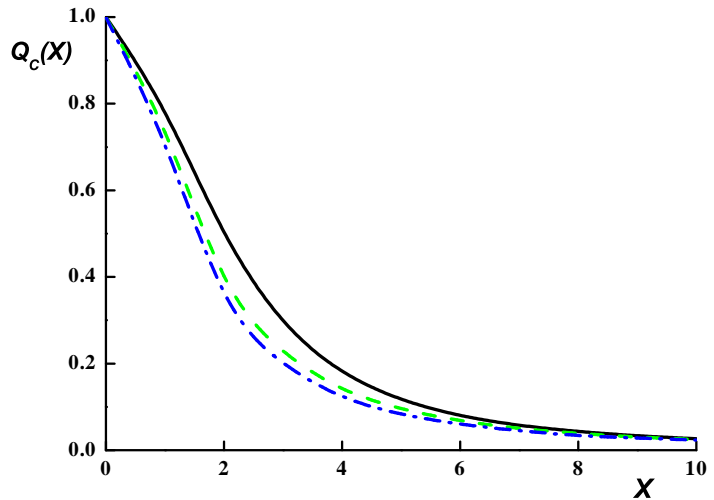

Figure 3. Quark correlator for different paths shown in Fig. 2. The solid line is for the straight-line path $(h=0)$; dashed line is for the broken-line path, with $h=3$; and dot-dashed line is for $h=6$. All the distances $(x, h)$ are in units of the instanton size $\rho$.

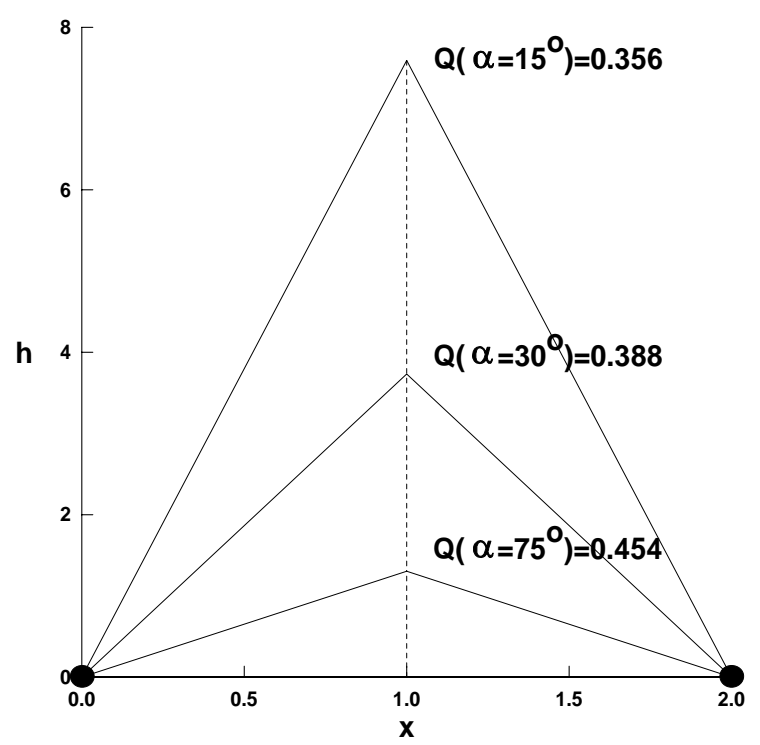

Figure 4. Different paths between quarks that are at fixed distance $x=2$, considering the same distance of the cusp from each quark. Indicated inside the figure we have three representative cases, with the corresponding values of the correlator. 


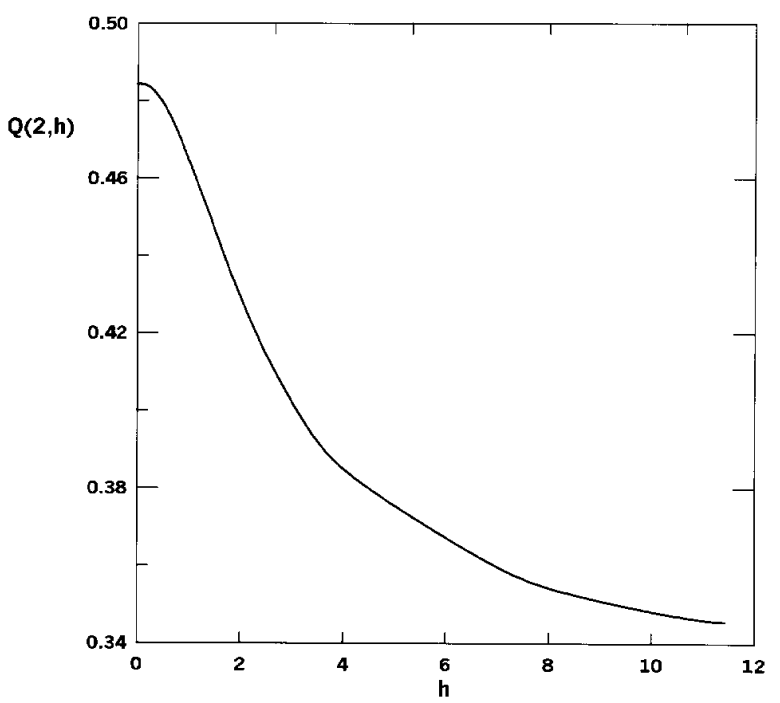

Figure 5. Quark correlator for different paths shown in Fig. 4, as a function of the cusp distance. The quarks are at fixed distance $x=2$ between them, and at the same distances from each cusp.

Finally, we observe that our main result is the strong dependence of the correlators on the shape of the path (Fig. 5), which can particularly be more relevant for the light quark propagation. The correlator corresponding to the straightline path, given in Eq. (3), has the largest signal (for every distance $h$ ), compared with other choices of the path (Fig. 3). Every deformation from the straight-line path produces a smaller value for the correlator.

\section{Acknowledgments}

Our thanks to Fundação de Amparo à Pesquisa do Estado de São Paulo (FAPESP), that makes possible this collaboration. L.T. also thanks the partial support received from Conselho Nacional de Desenvolvimento Científico e Tecnológico do Brasil. AED thanks for partial support from RFBR (Grants nos. 02-02-16194, 03-02-17291, 0402-16445), INTAS (Grant no. 00-00-366).

\section{References}

[1] S.V. Mikhailov, A.V. Radyushkin, Phys. Rev. D 45, 1754 (1992).

[2] A.E. Dorokhov and L. Tomio, Phys. Rev. D 62, 014016 (2000).

[3] B.M. Barbashov, Sov. Phys. JETP 21, 402 (1965).

[4] M. D'Elia, A. Di Giacomo, and E. Meggiolaro, Phys. Rev. D 59, 054503 (1999).

[5] A.E. Dorokhov, S. V. Esaibegian, and S. V. Mikhailov, Phys. Rev. D 56, 4062 (1997).

[6] A.E. Dorokhov, S. V. Esaibegian, A.E. Maximov, and S. V. Mikhailov, Eur. Phys. J. C 13, 331 (2000).

[7] A. Di Giacomo and E. Meggiolaro, Phys. Lett. B 537, 173 (2002).

[8] S.V. Mikhailov, Phys. Atom. Nucl. 56, 650 (1993) [Yad. Fiz. 56, 143 (1993)].

[9] A.G. Grozin, Int. J. Mod. Phys. A 10, 3497 (1995). 